\title{
The profile of meningitis in a tertiary paediatric hospital in South Africa
}

\author{
L Jansz, ${ }^{1}$ BSc; H Buys, ${ }^{2}$ FCP; M van Dijk, ${ }^{1} \mathrm{PhD}$; U Rohlwink, ${ }^{3} \mathrm{PhD}$

\begin{abstract}
${ }^{1}$ Department of Paediatric Surgery, Erasmus MC-Sophia Children's Hospital, Rotterdam, the Netherlands ${ }^{2}$ Department of Paediatrics and Child Health, Faculty of Health, University of Cape Town, and Red Cross War Memorial Children's Hospital, South Africa

${ }^{3}$ Division of Neurosurgery, Faculty of Health, University of Cape Town, and Red Cross War Memorial Children's Hospital, South Africa
\end{abstract}

Corresponding author: L Jansz (lucajansz@gmail.com)

\begin{abstract}
Background. Meningitis in children is a major health problem worldwide, leading to high rates of mortality and morbidity.
Objectives. To describe the profile of patients treated for meningitis at a leading tertiary paediatric hospital (Red Cross War Memorial Children's Hospital) in South Africa.

Methods. This study describes all patients treated for suspected meningitis at our hospital from 2010 to 2012. Data were retrospectively collected from patient folders.

Results. A total of 706 patients with meningitis were divided into definite bacterial $(n=42)$, probable bacterial $(n=113)$, partially treated bacterial $(n=100)$, viral $(n=412)$, and tuberculous meningitis (TBM, $n=39))$ infections. Fever $(74.7 \%)$, headache $(66.4 \%)$, vomiting $(52.1 \%)$ and irritability (34.5\%) were common symptoms in all patients; TBM patients presented more often with weight loss, neck stiffness, lethargy and abnormal neurological signs. Symptoms were usually present for 1 - 2 days in viral and bacterial meningitis, and 8 days in TBM. The median duration of hospitalisation was 1 day for viral meningitis, 2 days for all three groups of bacterial meningitis and 22 days for TBM, before referral to primary or secondary hospitals.

Conclusion. Patients with meningitis in this study often presented with nonspecific symptoms, making it difficult to clinically differentiate between types of meningitis. TBM patients presented more often with neurological fallout, and had a longer duration of symptoms. Patients often received antibiotics before a lumbar puncture was performed, further compounding the difficulty of diagnosis. Swift, sensitive and specific novel diagnostic tools would aid clinicians in making timeous and accurate diagnoses and treatment decisions.
\end{abstract}

S Afr J Child Health 2018;12(1):15-20. DOI:10.7196/SAJCH.2018.v12i1.1428

Meningitis is an major health problem worldwide, including in South Africa (SA), which has an annual incidence of 4 per 100000 cases in the general population, and it occurs commonly in infants (with an incidence of 40 per 100000 ). This incidence rate is probably an underestimate, because the rate of positive cerebrospinal fluid (CSF) culture results is low, and negative cultures are excluded in these numbers. ${ }^{[1]}$ Furthermore, owing to the rising incidence of tuberculosis in SA, the increasing number of patients with tuberculous meningitis (TBM) is of great concern. ${ }^{[2]}$ Symptoms of meningitis may be nonspecific, differ between age groups and have little diagnostic value. ${ }^{[3-5]}$ Presenting symptoms include fever, irritability, poor feeding, vomiting, seizures, headaches, photophobia and confusion. Older children ( $>3$ years) tend to present more similarly to adults, with classical signs such as headaches, neck stiffness and photophobia. ${ }^{[1]}$ Signs of meningeal irritation are present in $75 \%$ of children with bacterial meningitis. ${ }^{[5]}$ Seizures may occur in meningitis, but should not be confused with the common febrile seizures in the age range of 6 months - 6 years ${ }^{[4]}$ Fever occurs regularly, but is less common in infants. ${ }^{[3]}$ Since the diagnostic value of clinical features in children with meningitis is limited, a low threshold for the use of diagnostic tools such as lumbar puncture (LP) for suspected meningitis in infants and young children is recommended. ${ }^{[1,4]}$

Typical CSF findings for bacterial meningitis are an elevated white blood cell count (WCC), with polymorph predominance, decreased glucose and increased protein ${ }^{[6,7]}$ TBM is associated with elevated lymphocytes and protein; glucose may be decreased. ${ }^{[8]}$ Viral meningitis occurs with CSF findings closer to normal ranges, but a slightly elevated WCC is common ${ }^{[9]}$ Usually there is lymphocyte predominance, but early in the disease course, CSF may show polymorph predominance. ${ }^{[6,9]}$ Although CSF findings may confirm the presumptive diagnosis of meningitis, laboratory results are not always easy to interpret, since immediate administration of antibiotics in suspected meningitis is recommended in SA at primary care level, and CSF may be sterilised a few hours after antibiotics are given. ${ }^{[1,7]}$ Pretreatment with antibiotics is also associated with fewer positive CSF culture results, and affects CSF profiles, as glucose may increase and protein may decrease. ${ }^{[10]}$ Complete sterilisation may already occur within 2 - 4 hours after administering antibiotics. ${ }^{[1]}$ Furthermore, there are also discrepancies in the normal values used in different studies, and the CSF criteria used to distinguish between viral and bacterial aetiologies may also differ between studies.

Meningitis as a cause of death is more common in developing countries (24.0 deaths per 100000 children) than in the developed world (1.6 deaths per 100000 children), ${ }^{[12]}$ yet little has been published on paediatric meningitis in developing countries, where the profile of meningitis differs from that in the developed-world context. ${ }^{[13]}$ Previous studies have reported a decrease in meningitis caused by Haemophilus influenzae type B (Hib) since the introduction of the Hib vaccine; however, there has been an increase in paediatric TBM cases in the last 30 years in the Western Cape province of SA, and meningitis remains a major cause of death in SA children. ${ }^{[2,8,14,15]}$ The aim of this study is to describe the profile of patients treated for bacterial and viral meningitis at a tertiary paediatric hospital in SA.

\section{Methods}

All patients who underwent a diagnostic LP between 1 July 2010 and 30 June 2012 at the Red Cross War Memorial Children's Hospital (RCWMCH) were included for record review. Patients were identified using the laboratory database of all CSF analyses over this period. Records were thoroughly examined to identify all patients treated for meningitis. Normal values for CSF used by the hospital 
laboratory are described as there being no polymorphonucleocytes, $\leq 5$ lymphocytes, protein $\leq 0.8 \mathrm{~g} / \mathrm{L}$ or $\leq 0.45 \mathrm{~g} / \mathrm{L}$ (for $<1$ year of age or $>1$ year of age, respectively) and glucose $\geq 2.3 \mathrm{mmol} / \mathrm{L}$. Chloride values were not considered in categorising meningitis patients.

Patients were categorised as belonging to one of five types of meningitis. 'Viral meningitis' was diagnosed when CSF showed elevated WCC ( $>5$ lymphocytes, or any polymorphonucleocytes), with CSF chemistry (protein and glucose) within the normal range. Bacterial meningitis was divided into subgroups: 'definite bacterial meningitis', 'probable bacterial meningitis' and 'partially treated bacterial meningitis'. A definite bacterial meningitis categorisation was assigned when CSF culture or Gram stain was positive for a bacterial pathogen, 'probable bacterial meningitis' if CSF showed any polymorphonucleocytes in combination with elevated protein or decreased glucose, and 'partially treated bacterial meningitis' when a patient received antibiotics prior to LP, and CSF results showed predominantly polymorphonucleocytes, or elevated protein or decreased glucose. For the purposes of this study, the categorisation of TBM was based on a published consensus statement, which combines laboratory, clinical and radiological criteria. ${ }^{[16]}$ Patients falling into the 'definite' and 'probable TBM' groups were analysed as a single group. Uncategorised patients were defined as 'uncertain pathology' in the case of a normal WCC, but abnormal chemistry and no history of antibiotics prior to LP. 'Partially treated meningitis or misdiagnosed' was assigned when the patient received antibiotics prior to LP, but showed normal CSF, and 'misdiagnosed' when CSF was normal and there was no history of antibiotics prior to LP.

The CSF studies were processed by the National Health Laboratory Service, using standard laboratory methods. CSF was not routinely processed for viral culture or polymerase chain reaction (PCR). All CSF studies were processed for Gram stain and culture. In cases of suspected TBM, acid-fast stain was added.

\section{Data collection}

Demographic and clinical data, including history, presenting signs and symptoms, diagnostic and laboratory tests, treatment and inhospital death, were retrieved from patient folders. Outcome was not recorded, as the RCWMCH is a tertiary hospital, and most patients were referred to primary or secondary hospitals for further management after the initial diagnosis and stabilisation. Follow-up at other hospitals was beyond the reach of this study.

\section{Statistics}

Normally distributed variables were summarised using mean (standard deviation), and non-normally distributed variables as median (interquartile range (IQR)). Statistical comparisons between groups for continuous variables were performed using Kruskal-Wallis tests, and $\chi^{2}$ and Fisher's exact tests in the case of categorical variables.

This study was approved by the Human Research Ethics Committee of the University of Cape Town (ref. no. HREC 177/2012).

\section{Results}

Between 1 July 2010 and 30 June 2012, a total of 7410 LPs were performed in the RCWMCH, and 805 patients were treated for meningitis. The bacterial meningitis group consisted of 42 definite cases $(5.9 \%), 113$ probable cases $(16.0 \%)$ and 100 partially treated cases $(14.1 \%)$. The confirmed bacterial cases group contained 31 (73.8\%) positive CSF cultures, and another 11 (26.2\%) positive Gram stains. Cultured organisms $(n=31)$ were Streptococcus pneumonia ( $n=9 ; 29.0 \%)$, Group B Streptococcus $(n=6 ; 19.4 \%)$, Neisseria meningitides $(n=5 ; 16.1 \%)$, Haemophilus influenzae $(n=4$; $12.9 \%)$, Escherichia coli ( $n=2 ; 6.5 \%)$, Staphylococcus epidermidis $(n=2 ; 6.5 \%)$, Enterobacter cloacae complex $(n=1 ; 3.2 \%)$, Streptococcus pyogenes $(n=1 ; 3.2 \%)$ and a nontyphoidal Salmonella $(n=1 ; 3.2 \%)$. A total of 39 patients met the criteria for definite $(n=20)$ or probable
TBM ( $n=19)$ (5.5\%). Viral meningitis cases made up the largest group, with 412 cases $(58.4 \%)$. There were 99 patients who did not meet any of the meningitis criteria, and were categorised as 'possible TBM' $(n=36)$, 'uncertain pathology' $(n=9)$, 'partially treated/misdiagnosed' $(n=37)$ or 'misdiagnosed' $(n=17)$. These patients were not included in the results (see Fig. 1).

Table 1 gives an overview of the background characteristics for the total sample; Table 2 provides an overview on presenting symptoms, signs, diagnostics and treatment.

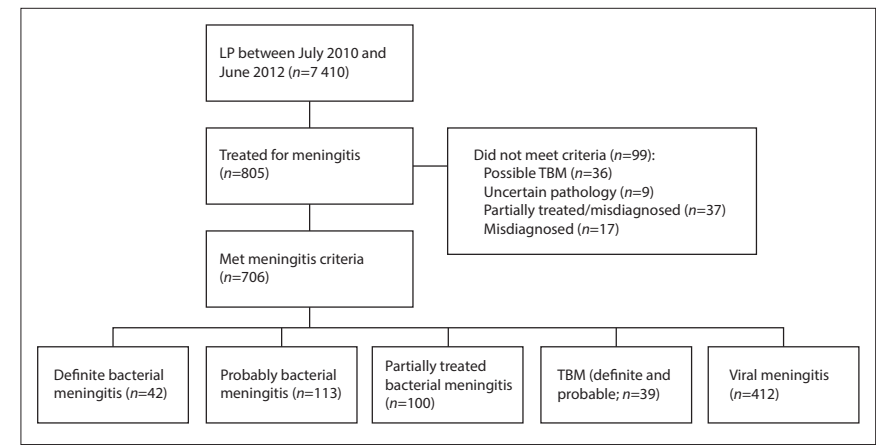

Fig. 1. Flowchart of patient inclusion and categorisation ( $L P=$ lumbar puncture; $T B M=$ tuberculous meningitis.)

Table 1. Demographic characteristics of patient population in children with meningitis $(N=706)$

\begin{tabular}{|c|c|}
\hline Demographic characteristic & $n(\%)$ \\
\hline \multicolumn{2}{|l|}{ Gender } \\
\hline Male & $436(61.8)$ \\
\hline Female & $270(38.2)$ \\
\hline \multicolumn{2}{|l|}{ Age group } \\
\hline Neonate (<1 month) & $39(5.5)$ \\
\hline 1 month - 1 year & $268(38.0)$ \\
\hline$>1$ year -6 years & $264(37.4)$ \\
\hline$>6$ years & $135(19.1)$ \\
\hline \multicolumn{2}{|l|}{ Language spoken at home } \\
\hline English & $296(41.9)$ \\
\hline IsiXhosa & $235(33.3)$ \\
\hline Afrikaans & $110(15.6)$ \\
\hline Other/unknown & $65(9.2)$ \\
\hline \multicolumn{2}{|l|}{ HIV status $(n=510)^{*}$} \\
\hline Positive & $50(9.8)$ \\
\hline HIV-exposed, PCR-negative & $66(12.9)$ \\
\hline 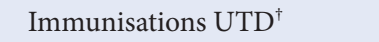 & $436(90.3)$ \\
\hline Previous meningitis ${ }^{\ddagger}$ & $20(3.2)$ \\
\hline \multicolumn{2}{|l|}{ Diagnosis } \\
\hline Bacterial (definite) & $42(5.9)$ \\
\hline Bacterial (probable) & $113(16.0)$ \\
\hline Bacterial (partially treated) & $100(14.2)$ \\
\hline TBM & $39(5.5)$ \\
\hline Viral & $412(58.4)$ \\
\hline In-hospital deaths & $16(2.3)$ \\
\hline \multicolumn{2}{|l|}{ Discharge destination ${ }^{\S}$} \\
\hline Home & $330(48.0)$ \\
\hline Other healthcare facility & $358(52.0)$ \\
\hline \multicolumn{2}{|c|}{ 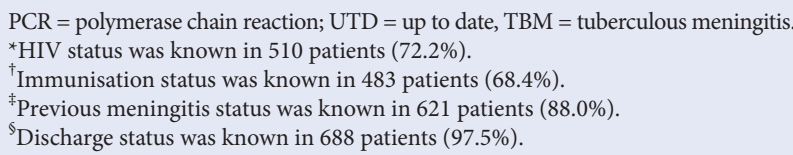 } \\
\hline
\end{tabular}


Table 2. Symptoms, signs, diagnostics and treatment in patient population in children with meningitis $(N=706)$

\begin{tabular}{|c|c|c|c|c|c|c|}
\hline & \multicolumn{6}{|c|}{ Meningitis type, $n(\%)$} \\
\hline & $\begin{array}{l}\text { Bacterial } \\
\text { (definite), } \\
n=42\end{array}$ & $\begin{array}{l}\text { Bacterial } \\
\text { (probable), } \\
n=113\end{array}$ & $\begin{array}{l}\text { Bacterial } \\
\text { (partially } \\
\text { treated), } \\
n=100\end{array}$ & TBM, $n=39$ & Viral, $n=412$ & $p$-value \\
\hline \multicolumn{7}{|l|}{ Symptom } \\
\hline Fever & $34(81.0)$ & $77(68.1)$ & $70(70.0)$ & $20(51.3)$ & $326(79.1)$ & $<0.001$ \\
\hline Vomiting & $12(28.6)$ & $55(48.7)$ & $50(50.0)$ & $20(51.3)$ & $230(55.8)$ & 0.012 \\
\hline Irritability & $20(47.6)$ & $38(33.6)$ & $38(38.0)$ & $10(25.6)$ & $137(33.3)$ & 0.265 \\
\hline Headache $(n=345)^{\star}$ & $3(33.3)$ & $22(40.7)$ & $28(65.1)$ & $14(50.0)$ & $162(76.8)$ & $<0.001$ \\
\hline Neck stiffness & $10(23.8)$ & $36(31.9)$ & $23(23.0)$ & $26(66.7)$ & $123(29.9)$ & $<0.001$ \\
\hline Loss of appetite & $26(61.9)$ & $27(23.9)$ & $24(24.0)$ & $17(43.6)$ & $122(29.6)$ & $<0.001$ \\
\hline Lethargy & $11(26.2)$ & $19(16.8)$ & $14(14.0)$ & $19(48.7)$ & $60(14.6)$ & $<0.001$ \\
\hline Seizures & $12(28.6)$ & $27(23.9)$ & $19(19.0)$ & $9(23.1)$ & $57(13.8)$ & 0.023 \\
\hline Failure to thrive & $2(4.8)$ & $17(15.0)$ & $10(10.0)$ & $18(46.2)$ & $27(6.6)$ & $<0.001$ \\
\hline Not walking $(n=399)^{\dagger}$ & $1(8.3)$ & $1(1.6)$ & $1(2.0)$ & $8(24.2)$ & $5(2.0)$ & $<0.001$ \\
\hline Duration of symptoms, (days; median (IQR)) & $2(1-3)$ & $1(1-3)$ & $1(1-2)$ & $8(4-15)$ & $1(1-2)$ & $<0.001$ \\
\hline \multicolumn{7}{|l|}{ Sign } \\
\hline GCS $<15(n=130)^{\ddagger}$ & $7(100.0)$ & $13(68.4)$ & $3(21.4)$ & $20(71.4)$ & $9(14.5)$ & $<0.001$ \\
\hline Focal neurology $(n=512)^{\S}$ & $11(32.4)$ & $19(21.8)$ & $6(9.2)$ & $24(64.9)$ & $21(7.3)$ & $<0.001$ \\
\hline Signs of raised ICP $(n=283)^{9}$ & $14(51.9)$ & $6(14.3)$ & $4(9.8)$ & $7(30.4)$ & $17(11.3)$ & $<0.001$ \\
\hline CN palsy $(n=59)^{\|}$ & $2(40.0)$ & $3(42.9)$ & $1(11.1)$ & $11(55.0)$ & $3(16.7)$ & 0.057 \\
\hline \multicolumn{7}{|l|}{ Diagnostic } \\
\hline LP before treatment & $19(45.2)$ & $51(45.1)$ & 0 & $11(28.2)$ & $287(69.7)$ & $<0.001$ \\
\hline Raised OP $\left(>25 \mathrm{~cm} \mathrm{H}_{2} \mathrm{O}\right)$ on first LP $(\mathrm{n}=41)^{\star *}$ & 0 & $3(30.0)$ & $1(33.3)$ & $10(55.6)$ & $1(14.3)$ & 0.209 \\
\hline CT brain & $19(45.2)$ & $37(32.7)$ & $17(17.0)$ & $39(100)$ & $44(10.7)$ & $<0.001$ \\
\hline CT before LP $(n=156)$ & $15(78.9)$ & $32(86.5)$ & $13(76.5)$ & $34(87.2)$ & $38(86.4)$ & 0.747 \\
\hline \multicolumn{7}{|l|}{ Treatment } \\
\hline Antibiotics (including pre-hospital) & $39(92.9)$ & $108(95.6)$ & $100(100)$ & $39(100)$ & $346(84.0)$ & $<0.001$ \\
\hline Prehospital antibiotics & $8(19.0)$ & $29(25.7)$ & $43(43.0)$ & $11(28.2)$ & 49 (11.9) & $<0.001$ \\
\hline Antibiotics duration (days, median (IQR)) & $7.5(5-14)$ & $7(5-10)$ & $7(5-7)$ & $7(7-9)$ & $5(2-7)$ & $<0.001$ \\
\hline Viral therapy & $10(23.8)$ & $23(20.4)$ & $9(9.0)$ & $9(23.1)$ & $22(5.3)$ & $<0.001$ \\
\hline Steroids & $16(38.1)$ & $23(20.4)$ & $5(5.0)$ & $36(92.3)$ & $14(3.4)$ & $<0.001$ \\
\hline Surgery & $3(7.1)$ & $3(2.7)$ & $3(3.0)$ & $26(66.7)$ & $2(0.5)$ & $<0.001$ \\
\hline EVD & $1(2.4)$ & $1(0.9)$ & $2(2.0)$ & $20(51.3)$ & $2(0.5)$ & \\
\hline Shunt & $1(2.4)$ & 0 & 0 & $19(48.7)$ & $1(0.2)$ & \\
\hline Hospitalisation (days; median (IQR)) & $2(1-10.5)$ & $2(1-9)$ & $2(1-4.5)$ & $22(12-35)$ & $1(1-2)$ & $<0.001$ \\
\hline 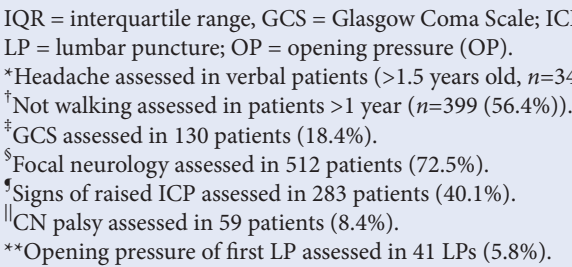 & $\begin{array}{l}\text { tracranial press } \\
8 \%))\end{array}$ & $\mathrm{CN}=$ cranial ne & ; $\mathrm{CT}=$ comp & omography; EVD & external ventricul & r drain; \\
\hline
\end{tabular}

The majority of the patients were male (61.8\%), and the median (IQR) age was $1.45(0.3-4.9)$ years. The age distribution was different between diagnosis groups $(p<0.001)$, ranging from a median (IQR) age of $0.46(0.1-1.1)$ years in the definite bacterial group, to a median (IQR) age of $3.1(1.3-5.2)$ years in TBM patients (Fig. 2). HIV infection was diagnosed in 50 (7.1\%) patients, $460(65.1 \%)$ patients were not infected, and in 196 (27.8\%) patients, HIV status was not tested, recorded or known. Most (75.1\%) patients had been referred from another health facility, and just over half $(52.0 \%)$ of the patients were discharged to another hospital after initial hospitalisation.

\section{Signs and symptoms}

Common presenting symptoms were fever ( $n=527 ; 74.7 \%)$, headache $(n=229 ; 66.4 \%)$, vomiting $(n=367 ; 52.0 \%)$ and irritability $(n=243$; $34.4 \%)$. Weight loss, neck stiffness, altered level of consciousness and lethargy were more common in TBM than in all other groups $(p<0.001)$. Fever was less common $(51.3 \%)$ in the TBM group than in the others $(68.1 \%-81.0 \%(p=0.001)$. Patients with TBM also had the longest duration of symptoms: the median (IQR) was 8 (4 - 15) days, compared with $2(1-3)$ days for definite bacterial meningitis, $1(1-3)$ day for probable bacterial meningitis and $1(1-2)$ day for partially treated bacterial and viral meningitis $(p<0.001)$ (Fig. 3$)$. 
Signs were not consistently recorded in patient records, with missing values ranging from $27.5 \%$ for focal neurology, up to $81.6 \%$ for Glasgow Coma Scale (GCS). A GCS $<15$ was more common in definite bacterial (100\%), probable bacterial (68.4\%) and TBM (71.4\%) than viral (14.5\%) and partially treated bacterial meningitis $(21.4 \%)(p<0.001)$. Neurological signs and symptoms of raised intracranial pressure (ICP) were common in definite bacterial meningitis and TBM.

\section{Diagnostics}

All patients in this study underwent one or more LPs. The majority (69.7\%) of patients with viral meningitis received their LP before antibiotic treatment, in contrast with TBM patients (28.2\%) $(p<0.001)$, who were more likely to be given antibiotics prior to LP.

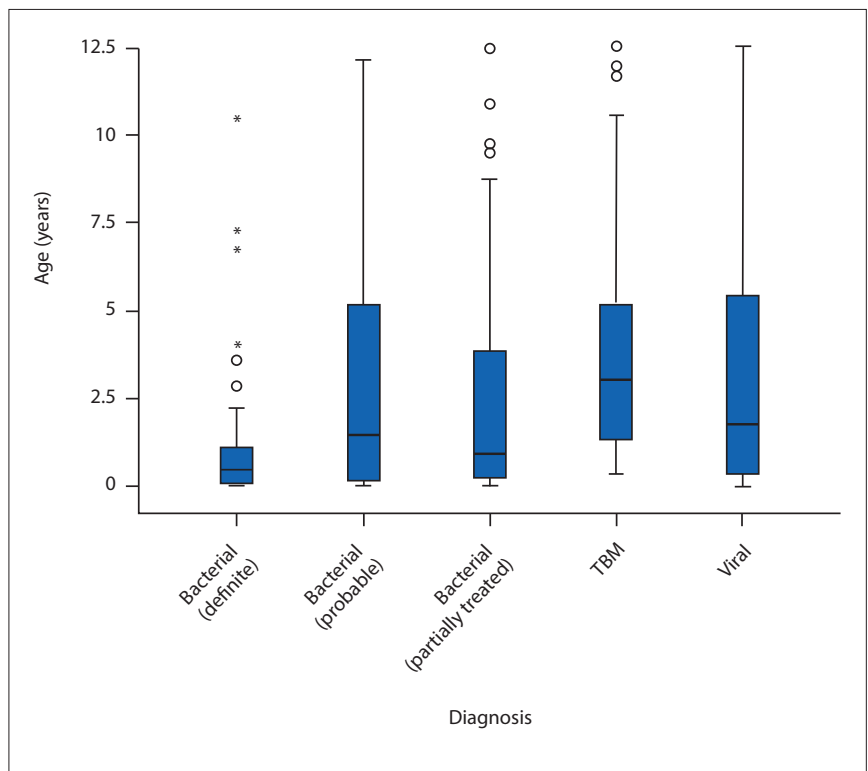

Fig. 2. Age distribution of meningitis patients according to diagnosis. $(T B M=$ tuberculous meningitis.$)$

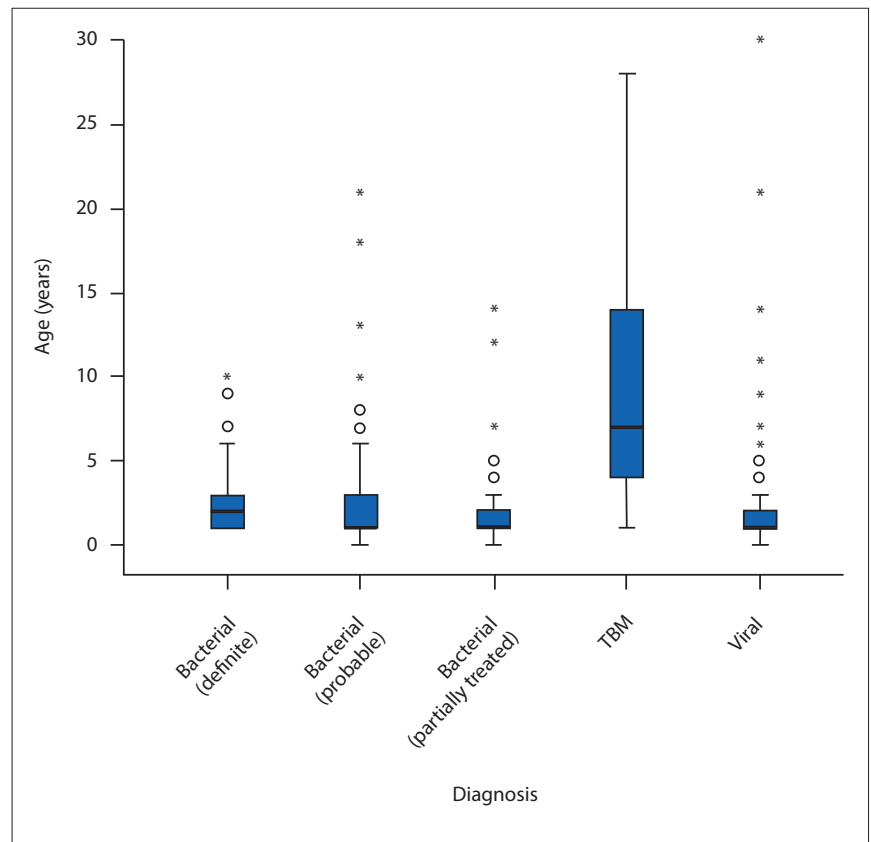

Fig. 3. Duration of symptoms in days at presentation in the hospital, according to diagnosis (not shown: 2 tuberculous meningitis (TBM) outliers ( $>2$ standard deviations at 49 and 60 days.)).
The opening pressure (OP) on the first LP was documented in only $41(5.8 \%)$ of all LPs, but more often in patients with TBM (46.2\%; $p<0.001)$. A raised $\mathrm{OP}>25 \mathrm{~cm} \mathrm{H}_{2} \mathrm{O}$ was present in $10 \mathrm{TBM}$ patients (55.6\% of documented OPs in TBM; $25.6 \%$ of all TBM patients). Of these 10 patients, 9 were diagnosed with hydrocephalus on head computed tomography (CT) scans.

\section{Treatment}

Antibiotics were given to the majority of patients, from $84.0 \%$ in the viral group to $100 \%$ in partially treated bacterial meningitis and TBM. The duration of antibiotics is defined as the number of days for which they were prescribed. The viral cohort received antibiotics for a median (IQR) duration of $5(2-7)$ days, which was $2-2.5$ days fewer than the other groups $(p<0.001)$. The duration of hospital stay varied between groups $(p<0.001)$. The median duration ranged from 1 (viral meningitis) to 22 (TBM) days (Fig. 4). After hospitalisation, $48.0 \%$ of all patients were sent home, and $52.0 \%$ to another healthcare facility. TBM patients were more often referred to another healthcare facility after initial hospitalisation than other groups $(p=0.006)$ - only $20.6 \%$ of TBM patients were sent home directly.

\section{Discussion}

In this study, 706 paediatric patients with meningitis were evaluated with respect to signs, symptoms, diagnostics and treatment. These patients may be a fair representation of the paediatric meningitis population of the Western Cape, as RCWMCH was the primary paediatric hospital in this area during the study period, and many children were referred from primary care units. This study showed that it was difficult to differentiate between bacterial and viral meningitis based on clinical symptoms, which could account for $84.0 \%$ of the patients with viral meningitis receiving antibiotics. However, the durations of antibiotics and of hospitalisation were shorter in the viral meningitis group compared with other groups, which might indicate cautious initial use of antibiotics and hospitalisation, with a cessation of antibiotics once the clinical picture had been fully reviewed. Given the threat of increasing drug resistance, faster reliable diagnostic tools for bacterial meningitis are urgently needed. Several studies are looking into promising diagnostic tools, such as procalcitonin in CSF, real-time PCR, multiplex PCR-based reverse line blot and matrix-assisted laser desorption/ionisation time of flight mass spectrometry. ${ }^{[17-20]}$

Interestingly, the viral meningitis group received the lowest amount of viral therapy. This might be explained by the use of

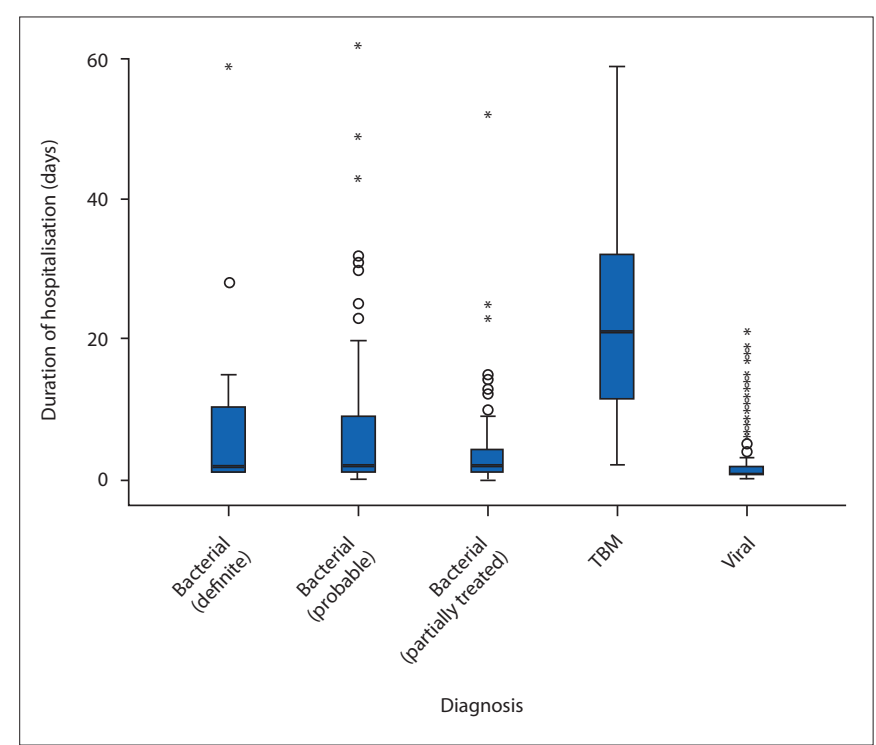

Fig. 4. Duration of hospitalisation in days, according to diagnosis (not shown: 2 tuberculous meningitis (TBM) outliers ( +1 standard deviation at 86 and 79 days.)). 
acyclovir (in addition to antibiotics) only when herpes simplex encephalitis was suspected or difficult to rule out immediately, or more likely because most viral meningitis cases are actually caused by enteroviruses, in which case acyclovir would not be indicated. ${ }^{[1]}$

Fever, headache, vomiting and irritability were common symptoms. However, these symptoms are not specific to meningitis. This is in accordance with previous studies that have demonstrated the limited diagnostic value of these symptoms in meningitis. ${ }^{[3,4,21]}$ More specific symptoms such as neck stiffness or focal neurology were less common; neck stiffness occurred in less than one-third of patients with viral $(29.9 \%)$ and bacterial meningitis $(23.0-31.9 \%)$. Focal neurology was present in only $7.3 \%$ in viral meningitis but went up to $32.4 \%$ in definite bacterial meningitis. Although bacterial and viral meningitis seemed to present quite similarly, TBM tended to present differently, with more severe and prolonged symptoms, such as weight loss, neck stiffness and an altered level of consciousness, in keeping with the more insidious nature of TBM. Given that clinical presentation and history form an important component of the presumptive diagnosis of this potentially devastating disease, the presence of these symptoms could alert clinicians to prioritise TBM in their differential diagnosis. Rapid diagnosis of TBM remains challenging, but research into novel diagnostic tests is on going. Some potential candidates include GeneXpert and the LAM-ELISA assay. GeneXpert is an additional test for CSF with reported sensitivity and specificity of $59.3 \%$ and $99.5 \%$, respectively. ${ }^{[22]}$ The test is fairly easy to operate, and provides results within 2 hours, making it a valuable asset for diagnosing TBM. ${ }^{[23]}$ LAM-ELISA is a urinary test, providing a simple diagnostic test for TBM, but its sensitivity is suboptimal. ${ }^{[24]}$

The difficulty in interpreting clinical signs and CSF results emphasises the need for better diagnostic tools, to avoid not only missing an important diagnosis, but also administering treatment unnecessarily, in the case of viral meningitis. Multiple clinical decision scores have been developed and validated, with sensitivities ranging from 0.83 to 1.00 , and specificity from 0.36 to $0.85 .{ }^{[25]}$ The bacterial meningitis score (BMS) is the most promising, with a sensitivity of $99.3 \%$ and specificity $62.1 \%$ in a recent meta-analysis. ${ }^{[26]}$ This suggests that the BMS could serve as a valuable additional tool in the clinical assessment of children with suspected meningitis, although some false positives would probably occur. The BMS consists of five criteria (CSF Gram stain positive, CSF protein $>0.8 \mathrm{~g} / \mathrm{L}$, blood absolute neutrophil count $>10000$ cells $/ \mathrm{mm}^{3}$, history of seizures during current illness and CSF neutrophil count $>1000$ cells $/ \mathrm{mm}^{3}$ ) to determine the risk of bacterial meningitis in children with CSF pleocytosis. Patients with a BMS of 0 points are likely to have aseptic meningitis, and can be considered for outpatient management. ${ }^{[26]}$

Tygerberg Hospital, another tertiary hospital in the Western Cape, has carried out 3 studies of its meningitis patients in the past 30 years. ${ }^{[2,27,28]}$ The median age of our study population (1.45 years) is similar to that found in the most recent Tygerberg study (1.4 years), and the majority of meningitis diagnoses were also of viral meningitis. ${ }^{[2]}$ However, the proportion of TBM patients was much higher in the Tygerberg study ( $22 \%$ v. $5.5 \%$ in this study). This might be explained by differences in the diagnostic protocols across the two hospitals, or it may be a reflection of time, as the Tygerberg study included patients over a 20 -year period, when TBM might have been more common.

HIV results were available in $72.2 \%$ of our patients, and the number of patients who tested positive was low $(9.8 \%$ of tested patients), which reflects favourably on SA's proactive mother-tochild antiretroviral roll-out programme.

The tertiary nature of our hospital is demonstrated by the large number of patients referred from other healthcare facilities $(75.1 \%)$ and discharged to another hospital (52.0\%). This referral pattern could explain why many patients had received antibiotics before they arrived at RCWMCH, where the diagnostic LP was performed.
The World Health Organization (WHO) Integrated Management of Childhood Illness approach is widely used in SA, and nurses at primary care facilities give an immediate single dose of ceftriaxone in the case of suspected meningitis, prior to referring the child to a hospital. ${ }^{[2]]}$ Therefore the interpretation of our CSF results is challenging, given that the CSF can be sterilised and parameters can normalise within hours after antibiotics have been started. This might have contributed to the low number of culture-positive CSF results ( $4.4 \%$ of all patients) that we found.

Most TBM patients had received treatment before having an LP. Firstly, this may be owing to strong concerns about poor outcomes of untreated TBM. Secondly, it may be due to the increased risk of raised ICP in TBM, which is a contraindication for LP. Therefore antibiotic treatment may be commenced before a head CT can be performed to identify the risk of non-communicating hydrocephalus and the safety of an LP. ${ }^{[8]}$ However, despite this risk, about one in six TBM patients was not scanned before an LP. This may be a result of uncertainty regarding the diagnosis, the absence of obvious clinical signs of raised ICP and poor access to a CT scanner. In this study population, $25.6 \%$ of TBM patients had a raised opening pressure on their LP, and $84.6 \%$ had hydrocephalus, which is similar to what has been found in previous studies. ${ }^{[8]}$

Two important limitations of this study are the subjectivity of defining the different meningitis groups, and the retrospective nature of this study. To our knowledge, there is no consensus in the literature regarding exact case definitions or cut-off points in CSF values for different meningitis aetiologies. For instance, bacterial meningitis usually shows polymorphonucleocyte predominance and viral meningitis shows lymphocytosis, but in early viral meningitis there may be polymorphonucleocyte predominance too. ${ }^{[6]}$ Probable bacterial meningitis has been described as $>5$ leucocytes in CSF, up to $>10$ leucocytes, combined with a positive blood culture. ${ }^{[1,2,10,11]}$ The WHO defines probable bacterial meningitis as suspected meningitis with turbid CSF, leucocytosis $>100$ or leucocytosis $>10$ combined with elevated protein or decreased glucose ${ }^{[30]}$ However, a case definition for viral meningitis is lacking, other than clinically suspected meningitis. Research definitions for viral meningitis range from any suspected case of meningitis where another cause has been ruled out, to CSF with predominantly lymphocytes and normal chemistry or confirmed viral pathogen using PCR..$^{[2,31]}$ Uniform case definitions for bacterial and viral meningitis without a confirmed pathogen would be beneficial for future research. Defining the type of meningitis uses a combination of clinical signs and symptoms and the supporting information from CSF analysis, and is prone to subjective interpretation by clinicians. This made it difficult to retrospectively define the type of meningitis, when treatment was given based on the discretion of the treating clinician, and where variability in management choices may exist among clinicians due to the lack of standardised guidelines for patient management. However, this is also indicative of the challenges facing paediatricians daily: which child with fever, vomiting and headache has meningitis, and which child does not? Moreover, which child with meningitis should receive antibiotics and which child can be safely sent home after a short hospitalisation?

Another limitation of this study is that some data from the patient folders were missing. Some patient folders could not be located, many folders lacked detailed information, and there was variability in the history-taking of the clinicians, and their notes on the physical examination. This might have led to an underestimation of signs and symptoms. To ensure complete notes on history and clinical examination, a patient-folder template would be very useful. A final limitation of this study is the lack of detailed outcome and follow-up data, as many patients were sent to other health facilities to complete their treatment. Important complications of meningitis such as hearing loss and other neurological outcomes were therefore 
not documented in this study. Designing a prospective study on signs, symptoms, diagnostics and treatment in patients with meningitis would be valuable, to document the thinking behind clinical decisions when treating patients. This could provide insight into the difficult process of clinically assessing acutely sick children.

Paediatric meningitis remains challenging to diagnose and treat, given the nonspecific nature of the signs and symptoms, the lack of rapid and reliable diagnostic tests and the high frequency of immediate antibiotic administration. Although progress has been made, and new diagnostic test candidates are being investigated, further research is needed. This study provides a baseline of meningitis at this paediatric institution, and the clinical and treatment data presented may inform future studies that prospectively address some of the challenges of managing and diagnosing these patients.

\section{Conclusion}

Patients with meningitis in this study often presented with nonspecific symptoms such as fever, headache, vomiting and irritability, which make it difficult to clinically differentiate between different types of meningitis. However, patients with TBM presented more often with neurological signs and symptoms and had a longer duration of symptoms. Most patients received antibiotics, including the patients with viral meningitis, often before the LP was performed, further compounding the difficulty of diagnosis. Swift, sensitive and specific novel diagnostic tools would aid clinicians in making timeous and accurate diagnoses and treatment decisions.

Acknowledgements. The authors thank Selma Jaspers for contributing to data collection for this study.

Author contributions. LS: data collection, analysis and interpretation, revising; HB: interpretation of data, revising; MvD and UL: study design, analysis and interpretation of data, revising

\section{Funding. None.}

Conflicts of interest. None.

1. Boyles TH, Bamford C, Bateman K, et al. Guidelines for the management of acute meningitis in children and adults in South Africa. South Afr J Epidemiol Infect 2013;28(1):5-15. https://doi.org/10.1080/10158782.2013.11441513

2. Wolzak NK, Cooke ML, Orth H, van Toorn R. The changing profile of pediatric meningitis at a referral centre in Cape Town, South Africa. J Trop Pediatr 2012;58(6):491-495. https://doi.org/10.1093\%2Ftropej\%2Ffms031

3. Best J, Hughes S. Evidence behind the WHO guidelines: Hospital care for children - what are the useful clinical features of bacterial meningitis found in infants and children? J Trop Pediatr 2008;54(2):83-86. https://doi. org/10.1093\%2Ftropej\%2Ffmn013

4. Curtis S, Stobart K, Vandermeer B, Simel DL, Klassen T. Clinical features suggestive of meningitis in children: A systematic review of prospective data Pediatrics 2010;126(5):952-960. https://doi.org/10.1542\%2Fpeds.2010-0277

5. Kim KS. Acute bacterial meningitis in infants and children. Lancet Infect Dis 2010;10(1):32-42. https://doi.org/10.1016\%2Fs1473-3099\%2809\%2970306-8

6. Negrini B, Kelleher KJ, Wald ER. Cerebrospinal fluid findings in aseptic versus bacterial meningitis. Pediatrics 2000;105(2):316-319. https://doi. org/10.1542\%2Fpeds.105.2.316

7. Tunkel AR, Hartman BJ, Kaplan SL, et al. Practice guidelines for the management of bacterial meningitis. Clin Infect Dis 2004;39(9):1267-1284. https://doi.org/10.1086\%2F425368

8. Van Well GT, Paes BF, Terwee CB, et al. Twenty years of pediatric tuberculous meningitis: A retrospective cohort study in the Western Cape of South Africa. Pediatrics 2009;123(1):e1-8. https://doi.org/10.1542\%2Fpeds.2008-1353

9. Logan SA, MacMahon E. Viral meningitis. BMJ 2008;336(7634):36-40. https:// doi.org/10.1136\%2Fbmj.39409.673657.ae

10. Nigrovic LE, Malley R, Macias CG, et al. Effect of antibiotic pretreatment on cerebrospinal fluid profiles of children with bacterial meningitis. Pediatrics 2008;122(4):726-730. https://doi.org/10.1542\%2Fpeds.2007-3275
11. Kanegaye JT, Soliemanzadeh P, Bradley JS. Lumbar puncture in pediatric bacterial meningitis: Defining the time interval for recovery of cerebrospinal fluid pathogens after parenteral antibiotic pretreatment. Pediatrics 2001;108(5):1169-1174

12. Whiteford HA, Degenhardt L, Rehm J, et al. Global burden of disease attributable to mental and substance use disorders: Findings from the Global Burden of Disease study 2010. Lancet 2013;382(9904):1575-1586. https://doi.or g/10.1016\%2Fs0140-6736\%2813\%2961611-6

13. Furyk J, Swann O, Molyneux E. Systematic review: Neonatal meningitis in the developing world. Trop Med Internat Health 2011;16(6):672-679. https://doi. org/10.1111\%2Fj.1365-3156.2011.02750.x

14. Ntuli ST, Malangu N, Alberts M. Causes of deaths in children under five years old at a tertiary hospital in Limpopo province of South Africa. Glob J Health Sci 2013;5(3):95-100. https://doi.org/10.5539\%2Fgjhs.v5n3p95

15. Von Gottberg A, de Gouveia L, Madhi S, et al. Impact of conjugate Haemophilus influenzae type b (Hib) vaccine introduction in South Africa. Bull World Health Organ 2006;84(10):811-818. https://doi.org/10.2471\%2Fblt.06.030361

16. Marais S, Thwaites G, Schoeman JF, et al. Tuberculous meningitis: A uniform case definition for use in clinical research. Lancet Inf Dis 2010;10(11):803-812. https://doi.org/10.1016\%2Fs1473-3099\%2810\%2970138-9

17. Konstantinidis T, Cassimos D, Gioka T, et al. Can procalcitonin in cerebrospinal fluid be a diagnostic tool for meningitis? J Clin Lab Anal 2015;29(3):169-174. https://doi.org/10.1002/jcla.21746

18. Khumalo J, Nicol M, Hardie D, Muloiwa R, Mteshana P, Bamford C. Diagnostic accuracy of two multiplex real-time polymerase chain reaction assays for the diagnosis of meningitis in children in a resource-limited setting. PLOS ONE 2017;12(3):e0173948. https://doi.org/10.1371/journal.pone.0173948

19. Wang Y, Guo G, Wang $\mathrm{H}$, et al. Comparative study of bacteriological culture and real-time fluorescence quantitative PCR (RT-PCR) and multiplex PCRbased reverse line blot (mPCR/RLB) hybridization assay in the diagnosis of bacterial neonatal meningitis. BMC Pediatr 2014;14(1):224-231. https://doi org/10.1186/1471-2431-14-224

20. Segawa S, Sawai S, Murata S, et al. Direct application of MALDI-TOF mass spectrometry to cerebrospinal fluid for rapid pathogen identification in a patient with bacterial meningitis. Clinica Chimica Acta 2014;435:59-61. https:// doi.org/10.1016/j.cca.2014.04.024

21. Amarilyo G, Alper A, Ben-Tov A, Grisaru-Soen G. Diagnostic accuracy of clinical symptoms and signs in children with meningitis. Pediatr Emerg Care 2011;27(3):196-199. https://doi.org/10.1097\%2Fpec.0b013e31820d6543

22. Nhu NT, Heemskerk D, Thu DDA, et al. Evaluation of GeneXpert MTB/RIF for diagnosis of tuberculous meningitis. J Clin Microbiol 2014;52(1):226-233. https://doi.org/10.1128/jcm.01834-13

23. Lawn SD, Nicol MP. Xpert MTB/RIF assay: Development, evaluation and implementation of a new rapid molecular diagnostic for tuberculosis and rifampicin resistance. Fut Microbiol 2011;6(9):1067-1082. https://doi. org/10.2217/fmb.11.84

24. Minion J, Leung E, Talbot E, Dheda K, Pai M, Menzies D. Diagnosing tuberculosis with urine lipoarabinomannan: Systematic review and meta-analysis. Eur Respir J 2011;38(6):1398-1405. https://doi.org/10.1183/09031936.00025711

25. Kulik DM, Uleryk EM, Maguire JL. Does this child have bacterial meningitis? A systematic review of clinical prediction rules for children with suspected bacterial meningitis. J Emerg Med 2013;45(4):508-519. https://doi. org/10.1016\%2Fj.jemermed.2013.03.042

26. Nigrovic LE, Malley R, Kuppermann N. Meta-analysis of bacterial meningitis score validation studies. Arch Dis Child 2012;97(9):799-805. https://doi.org/10 .1136\%2Farchdischild-2012-301798

27. Donald P, Burger P, Becker W. Paediatric meningitis in the Western Cape. A 3-year hospital-based prospective survey. S Afr Med J 1986;70(7):391-395.

28. Donald PR, Cotton MF, Hendricks MK, Schaaf HS, de Villiers JN, Willemse TE. Pediatric meningitis in the Western Cape Province of South Africa. J Trop Pediatr 1996;42(5):256-261. https://doi.org/10.1093\%2Ftropej\% 2F42.5.256

29. Kerry T. A review of integrated management of childhood illness (IMCI). S Afr Fam Pract 2005;47(8):32-38. https://doi.org/10.1080\% 2F20786204.2005.10873272

30. WHO Coordinated Invasive Bacterial Vaccine Preventable Diseases (IB-VPD) Surveillance Network [updated January 2012]. Geneva: WHO, 2012. http:// www.who.int/immunization/monitoring_surveillance/resources/IB-VPD Case Defs.pdf (accessed 31 May 2017)

31. Hristea A, Olaru I, Baicus C, Moroti R, Arama V, Ion M. Clinical prediction rule for differentiating tuberculous from viral meningitis. Int J Tuberculosis Lung Dis 2012;16(6):793-798. https://doi.org/10.5588/ijtld.11.0687

Accepted 19 September 2017. 